\title{
Structures and Buildings: Referees 2019
}

The following is a list of referees who have reviewed papers for Structures and Buildings between 1 December 2018 and 18 November 2019. The Institution of Civil Engineers is very grateful for their assistance.

We are continually looking for suitable reviewers for papers submitted to Structures and Buildings. Papers published in the Proceedings of the ICE must be submitted to at least two independent referees to judge accuracy, style, impact, importance and interest.

\section{Ali Abbas}

Muthanna Abbu

Hany Abdalla

Shehata Abdel Raheem

Magd Abdel Wahab

Yaser Abdel-Aty

Adel Abdelnaby

Gholamreza Abdollahzadeh

Farid Abed

Daniel Abrams

Dimitra Achillopoulou

Jose Adam

Hamdy Afefy

Anoushiravan Afzali

Eleftherios Aggelopoulos

Stefano Agnetti

Masoud Ahmadi

Munir Ahmed

Tauqir Ahmed

Arash Akbari Hamed

Inci Akdeniz

Farhad Akhoundi

Vahid Akrami

Shahlaa Al Wakeel

Nabil Al-Akhras

M. Shahria Alam

Mohammed Alansari

Juan Aleman

Majid Ali

Saeid Alizadeh

Tarek Alqado

Adnan Al-Sibahy

Claudio Amadio

Giuseppina Amato

Jairo Andrade

Yukari Aoki

Hassan Aoude

Ricardo Araujo

Hector Archila

Mário Arruda

Musa Arslan

Ashraf Ashour

Mahmud Ashraf

Farhad Aslani

Panagiotis Asteris

Vegard Aune

Ewa Błazik-Borowa

Richard Bailey

Jeno Balogh

Richard Bartlett

Mike Bather
Mahesan Bavan

Robert Beale

Idris Bedirhanoglu

Chiara Bedon

Gopal Behera

Behrouz Behnam

Farhad Behnamfar

Elisa Bertolesi

Michele Betti

Rudraprasad Bhattacharyya

Fabio Biondini

Luke Bisby

Alan Bloodworth

Dan V Bompa

G Boscato

Steven Boyd

Mark A Bradford

Jorge Branco

David Bru

Emanuele Brunesi

Manuel Buitrago

Rodrigo Burgos

Chris J Burgoyne

Jose Cabrero

Pierfrancesco Cacciola

Qinlin Cai

Alfredo Camara

Rui Camposinhos

Daniel Cardoso

Daniele Casagrande

Claudia Casapulla

Fabio Casciati

Carlo Castiglioni

Rafael Castro-Triguero

Liborio Cavaleri

Murude Celikag

Fatih Cetisli

Tak-Ming Chan

Burachat Chatveera

Qing Chun

Antoni Cladera

David Collings

Pierluigi Colombi

Michael Constantinou

Stanko Coric

Marco Corradi

Alexandre A. Costa

Demetrios Cotsovos

Lee Cunningham

Elisabeth Da Nobrega Tavares

Bernadino D'Amico

If you are interested in reviewing articles on any topic related to engineering history and heritage, please submit your name, qualifications or CV, and areas of expertise. We are in need of individuals who will agree to review papers in a timely fashion (within 3 to 4 weeks of receipt) and provide confidential feedback to the Editorial Advisory Panel concerning the quality of the paper and any suggested revisions that would be appropriate.

If you are such a person, please contact Agnes Alvite (tel.: +44 (0)207 665 2204; e-mail: agnes.alvite@icepublishing.uk) for more information on the referee process.

Mario D'Aniello

Marija Danilovic

Antony Darby

Ana De Diego

Gianfranco De Matteis

Alexandre Del Savio

Diego Dellatorre

Hossein Derakhshan

Luigi Di Sarno

Hesham Diab

Alfredo Dias

Rossana Dimitri

Mizan Doğan

James Dow

Yingang Du

Antonio Duarte

Mahmud Dwaikat

Parviz Ebadi

Usama Ebead

Ahmed Elkady

Sameh El-Refaie

Germán Ercolani

Elif Erdil

Esmaeel Esmaeeli

Burak Evirgen

Beatrice Faggiano

Wei Fan

Alireza Faroughi

Fernando Ferreira

Pam Fom

Mohammad Fouad

Michalis Fragiadakis

Fabio Freddi

Feng Fu

Charis Gantes

Natalino Gattesco

SeyedAli Ghahari

Masoud Ghahremannejad

Renato Giannini

Amir Gilani

Ivan Giongo

Ana Girao Coelho

Michaela Gkantou

Francesco Graziotti

Minsheng Guan

Alessandra Gubana

Lotfi Guizani

Lanhui Guo

M N Habeeb

Abdeldayem Hadhood

Muhammad Hadi
Marijana Hadzima-Nyarko

Waleed Hamad

Mohammad Amin Hariri-Ardebili Kent Harries

Richard Harris

Rami Haweeleh

Stephen Hicks

Brian Hobbs

Ata Hojatkashani

Amir Horr

Seyed Abbas Hosseini

Neil Hoult

Zhaohui Huang

Yijie Huang

Abd Nacer Touati Ihaddoudene

Alper Ilki

Nicola Impollonia

Jason Ingham

Morteza Jamshidi

$\mathrm{S}$ Jayasree

Robert Jockwer

Stephen Jones

Cesar Juarez-Alvarado

Sakdirat Kaewunruen

Babak Kamrani Moghaddam

Elif Çagda Kandemir Mazanoglu

Theodore Karavasilis

Chris Karayannis

Hanuma Kasagani

Bohumil Kasal

Siavash Kazerani

Alexandra Keller

M lqbal Khan

Amar Khennane

Hyung-Joon Kim

Jochen Kohler

Katakalos Konstantinos

Lampros Koutas

Frank Kupferle

Albert Kwan

Michael Kyakula

Nicholas Kyriakides

Nelson Lam

Dennis Lam

Andreas Lampropoulos

John Lane

Denvid Lau

Moussa Leblouba

Daniel Sang-Hoon Lee

Chin-Long Lee

Deuck Hang Lee 


Wei Li
Bing Li
Fangyuan LI
Jianzhong Li
Carmine Lima
Ping Liu
Sergio Lopes
Herwing Lopez-Calvo
Paulo Lourenço
Christian Málaga-Chuquitaype
Riccardo Maddalena
Hany Madkour
Mahir Mahmod
Parthasarathi Mandal
Justin Marshall
Enzo Martinelli
Stuart Matthews
Edward Maunder
Sameh Mehanny
Behnam Mehrparvar
Elena Mele
Giovanni Minafã 2
Stergios Mitoulis
Amir Mofidi
Christopher Morley
Marius Mosoarca
Sherif Mourad
Joshua Mudie
Paul Mullett
Mohammadali Nahvinia

Roberto Nascimbene
Piotr Nazarko
Joao Negrao
David Nethercot
Themistoklis Nikolaidis
Ehsan Noroozinejad Farsangi
Emad Norouzzadeh Tochaei
Obeid Obeid
Fin O'Flaherty
Denio Ramam Oliveira
Eva Oller
Luciano Ombres
J Owen
Masoud Paknahad
Yuxin Pan
Maria Parisi
John Parker
Davorin Penava
Javier Pereiro-Barcelo
Adam Phillips
Kypros Pilakoutas
Vincenzo Piluso
Xudong Qian
Wai-Meng (Stephen) Quach
Imran Rafiq
Vlatka Rajcic
Nikhil Raut
Ermes Rizzi
Monique Rodrigues
Theodoros Rousakis

Juan Sagaseta
Yusa Sahin
Messaoud Saidani
Kay-Uwe Schober
Roberto Scotta
Junwon Seo
Andreea Serbescu
Ibrahim Shaaban
Ahmed Shaat
Rabee Shamass
Akanshu Sharma
MD Sheikh
Kshitij Shrestha
Sasan Siavashi
Karol Sikora
Bruna Silva
Pedro Simao
Marta Sitek
Andrew Smith
Romain Sousa
Nafsika Stavridou
Meini Su
RKL Su
Wei Sun
Muhammad Fiaz Tahir
D Tann
Thomas Tannert
Mu-Xuan Tao
Zhong Tao
Chiara Tardini

Waleed Thanoon

Marios Theofanous

Georgia Thermou

Roberto Tomasi

Cem Topkaya

Davide Trutalli

Themistoklis Tsalkatidis

Enrico Tubaldi

Nikolaos Tziavos

B Uy

Phillip Visintin

Prabhanjan Wagh

Baisheng Wang

Xuanding Wang

Bob Watkins

Chris Williams

Samuel Wong

Ping Xiang

Xu Xie

Bin Xu

Gürkan Yıldırım

Hua YingYu

Seyed Mehdi Zahrai

Ali Zare Hosseinzadeh

Binsheng Zhang

Yu Zheng

Wei Zhou

Xingang Zhou

Jue Zhu

WeiQing Zhu 